\title{
Quark-binding string*
}

\author{
S.-H. H. Tye \\ Stanford Linear Accelerator Center, Stanford University, Stanford, California 94305
}

(Received 11 December 1975)

\begin{abstract}
A relativistic invariant extended model of hadrons is constructed. The finite region where quarks can move freely is a line in a three-dimensional space. Physically, the construction resembles that of the MIT bag and the SLAC bubble. In the absence of quarks, the model reduces to the relativistic massless-string model. We then solve explicitly the closed-string version where massless quarks move only in the clockwise direction. The resulting quantum system does not satisfy Lorentz algebra. However, this negative result indicates that for an open (or closed) string, the quantum system is consistent if there are 22 quark fields. A quark-confining string is also constructed and discussed. This is obtained as an improved version of the quark-binding string. It incorporates many physically desirable features.
\end{abstract}

\section{INTRODUCTION}

Numerous phenomenological successes of the dual resonance amplitudes have stimulated the search for the physical picture underlying the model. Nambu and others ${ }^{1}$ observed that the spectrum of the Veneziano model ${ }^{2}$ is identical to that of a vibrating string. Subsequently the free-string formalism has been studied in detail, starting from an action written in terms of the area swept out by the string. ${ }^{3}$ More recently, the string picture has also emerged in many different investigations, ${ }^{4}$ and its relevance to hadron physics is generally accepted. Of course, the string picture is, at best, an approximate description of hadron physics. Its significance lies at least partly in the fact that it can be explicitly solved. This allows one to calculate its dynamical and static properties for comparison with experiments, starting from the exact quantum relativistic solution of the string; scattering ${ }^{5}$ and other interactions can be introduced as perturbations around the free-string solution, maintaining quantum mechanics and relativity at every order of perturbation. This approach toward hadron physics is attractive since it is the only approach we have experience in, namely, quantum electrodynamics; there the equations for the free photon and electron are first solved completely, and the electromagnetic interaction is then introduced as a perturbation around the exact solutions. Whether this gives an approximate description of hadrons we can tell only after the program has been carried out completely, beyond the free-string version.

The present string formalism has a few unsatisfactory features. Among them are the following: (1) The quantized string exists only in 26-dimensional Minkowski space. (2) There is a lack of incorporation of quarks (i.e., Dirac particles) and (maybe) their confinement. Numerous attempts have been made to overcome one or both of these shortcomings. This work may be considered as the first step of yet another attempt to improve the present string formalism. The formulation is motivated purely by the physical picture.

We construct the system of a string upon which physical quarks are confined. (By "physical" we mean the quarks are spinors in the four-dimensional Minkowski space but behave as scalars under a conformal transformation of the parameters describing the string.) We then proceed to solve the closed string along which massless quarks move around it in either the clockwise or anticlockwise direction. The mathematical formulation of this is summarized in Table I. The resulting quantized system does not satisfy Lorentz covariance, for the same reason as the Nambu string. However, the results do indicate that the complete system (with quarks moving both clock-

TABLE I. Equations of motion for the quark-binding string and its subsystem. Proper boundary conditions have to be included for an open or a closed string. The constraints need not be independent. The subsystem describes massless quarks moving clockwise around the closed string.

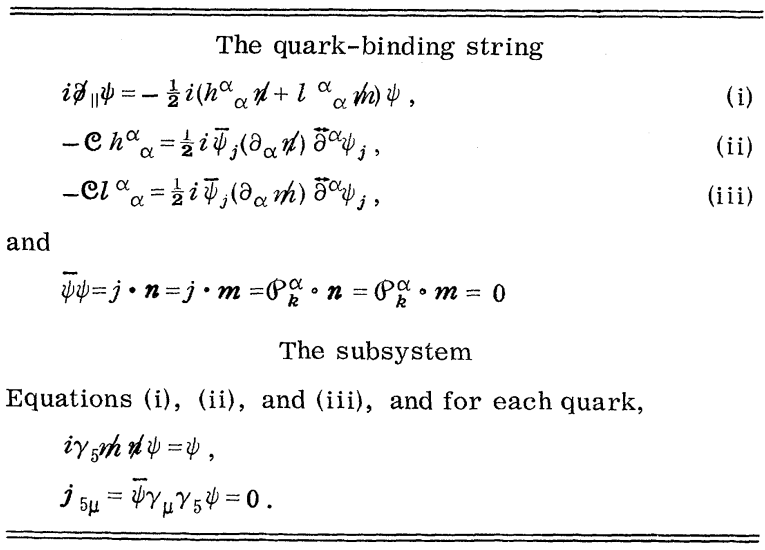


wise and anticlockwise around a closed string, or for an open string) will have Lorentz covariance if the number of quarks is 22. This speculation will be formulated as a conjecture.

Our string formalism is modeled after that of the MIT bag ${ }^{6}$ and the SLAC bubble, ${ }^{7}$ and the quantization procedure is modeled after that of the free string $^{3}$ and of the SLAC bubble in three-dimensional Minkowski space ${ }^{8}$ (which is equivalent to a quark-binding closed string).

From the investigation of the quark-binding string we find it has a few undesirable features: Its ground state is an empty string state; it also has exotic states, i.e., states with any number of quark excitation modes. To remove these bad features, a quark-confining string is constructed. Here the quarks come in color triplets interacting with color SU(3) gluon fields. Because we demand there be no local color electric field pointing out of the string, only color-singlet states exist. There are no gluon degrees of freedom; only color electric fields are left. Also there are no pure string modes, so that the ground state of this string is a meson; the quarks and the antiquarks may have masses, in which case they sit at the ends of the string, one at each end, linked by the appropriate electric flux line, which also defines the string.

Section II contains the formalism. In Sec. III we solve the closed string problem with only clockwise-moving quarks. In Sec. IV we examine the quantized system, and discover that Lorentz covariance is not satisfied. Section $V$ contains discussions and speculations. Here we speculate what the spectrum for the quark-binding string would look like. (By "quark-binding" we mean the absence of massless free pointlike quarks.) However, a string with any number of quark and antiquark modes can be present. In this section we also discuss how a quark-confining string can be written down in the action formalism. (By "quark confining" we mean only color-singlet states exist.) Some of the technical details and supplementary discussions are relegated to the four appendixes.

\section{FORMALISM}

The geometry of a relativistic string is that of a two-dimensional subspace $\left(V_{2}\right)$ embedded in a four-dimensional flat Minkowski space $\left(S_{4}\right)$. To describe a quark-binding string we must develop a convenient set of variables in the language of differential geometry. ${ }^{9}$

Let $x_{\mu}$ and $u^{\alpha}$ be the coordinates in the Minkowski space $S_{4}$ [with signature $(1,-1,-1,-1)$ ] and the curved space $V_{2}$ [with signature $\left.(1,-1)\right]$, respectively. The shape and position of the string are described by (see Fig. 1)

$$
x_{\mu}=x_{\mu}\left(u^{\alpha}\right), \quad \mu=0,1,2,3, \quad \alpha=0,1
$$

where the choice of the parameters $u^{\alpha}$ is arbitrary. We assume that $x_{\mu}$ is differentiable. (Our notation will be such that the indices $\alpha, \beta, \delta, \gamma$ refer to the $V_{2}$ space and $\mu, \nu, \lambda, \rho$ refer to the $S_{4}$ space.) The quantity

$$
\tau_{\alpha}^{\mu} \equiv \frac{\partial x^{\mu}}{\partial u^{\alpha}} \equiv x_{\mid \alpha}^{\mu} \equiv \partial_{\alpha} x^{\mu}
$$

is tangential to the string and is a vector in both $V_{2}$ and $S_{4}$. The induced metric for the $V_{2}$ space is given by

$$
g_{\alpha \beta}=\tau_{\alpha}^{\mu} \tau_{\beta \mu}=\tau_{\alpha} \cdot \tau_{\beta},
$$

which is a covariant symmetric tensor with signature $(1,-1)$.

At each point on the string, a plane perpendicular to the tangent vectors can be defined,

$$
U_{\mu \nu}=\frac{\epsilon_{\mu \nu \rho \lambda} \tau_{\alpha}^{\rho} \tau_{\beta}^{\lambda}}{\sqrt{-g}}, \quad \alpha \neq \beta
$$

where $\epsilon_{\mu \nu \rho \lambda}$ is totally antisymmetric, with $\epsilon_{0123}$ $=1$, and $g=\operatorname{det}\left(g_{\alpha \beta}\right)=g_{00} g_{11}-g_{01}{ }^{2}$. An alternative way to define this plane is by introducing two spacelike outward normals $n_{\mu}\left(u^{\alpha}\right)$ and $m_{\mu}\left(u^{\alpha}\right)$ :

$$
m \cdot \tau_{\alpha}=n \cdot \tau_{\alpha}=0 .
$$

They are normalized to unity,

$$
m_{\mu} m^{\mu}=m^{2}=n^{2}=-1,
$$

and, for convenience, are taken to be perpendicular to each other,

$$
n \cdot m=0 \text {. }
$$

Now $U_{\mu \nu}$ can be defined including its orientation in terms of $n_{\mu}, m_{\mu}$,

$$
U_{\mu \nu}=n_{\mu} m_{\nu}-m_{\mu} n_{\nu} .
$$

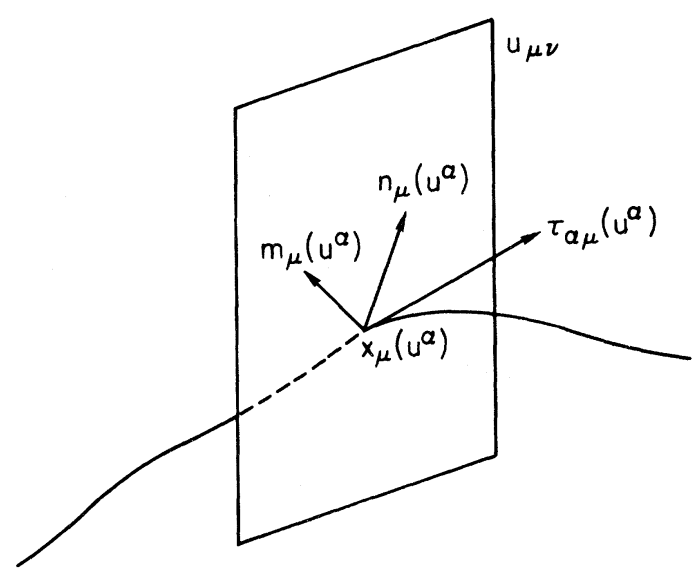

FIG. 1. The geometric coordinates of the string. 
The Minkowski metric at each point on the string can now be expressed in terms of the curvilinear coordinates provided by the set of linearly independent vectors,

$$
\eta_{\mu \nu}=\tau_{\mu}^{\alpha} \tau_{\alpha \nu}-n_{\mu} n_{\nu}-m_{\mu} m_{\nu},
$$

where $\tau_{\mu}^{\alpha}=g^{\alpha \beta} \tau_{\beta \mu} ; g^{\alpha \beta}$ is the inverse of $g_{\alpha \beta}$, and is a contravariant symmetric tensor. The tangential components of any vector $v_{\mu}$ can be projected out, $v_{\| \mu}=\left(v \cdot \tau^{\alpha}\right) \tau_{\alpha \mu}=v^{\alpha} \tau_{\alpha \mu}$.

Let us introduce some more quantities that are needed later. The derivatives of the normals

$$
\begin{aligned}
& n_{\mu \mid \alpha}=h_{\alpha \beta} \tau_{\mu}^{\beta}+\nu_{\alpha} m_{\mu}, \\
& m_{\mu \mid \alpha}=l_{\alpha \beta} \tau_{\mu}^{\beta}-\nu_{\alpha} n_{\mu}
\end{aligned}
$$

introduce the torsion $\nu_{\alpha}=n^{\mu}\left(m_{\mu \mid \alpha}\right)=-m^{\mu}\left(n_{\mu \mid \alpha}\right)$ and the symmetric curvature tensors

$$
\begin{aligned}
& h_{\alpha \beta}=n_{\mid \alpha} \cdot \tau_{\beta}=-n \cdot \tau_{\alpha \mid \beta}=-n \cdot \tau_{\beta \mid \alpha}=-n \cdot \tau_{\beta \| \alpha}, \\
& l_{\alpha \beta}=-m \cdot \tau_{\alpha \| \beta},
\end{aligned}
$$

where the covariant derivative of $\tau_{\alpha}^{\mu}$ is given by

$$
\begin{aligned}
\tau_{\alpha \| B_{B}}^{\mu} & =\tau_{\alpha \mid \beta_{\beta}}^{\mu}-\left\{{ }_{\alpha}{ }_{\beta}\right\} \tau_{\gamma}^{\mu} \\
& =h_{\alpha \beta} n^{\mu}+l_{\alpha \beta} m^{\mu} .
\end{aligned}
$$

The "Christoffel symbol of the second kind" i: function of the metric

$$
\begin{aligned}
\left\{\alpha_{\beta}{ }_{\beta}\right\} & =\frac{1}{2} g^{\gamma \delta}\left(g_{\alpha \delta \mid \beta}+g_{\delta \beta \mid \alpha}-g_{\alpha \beta \mid \delta}\right) \\
& =\tau^{\gamma} \cdot \tau_{\alpha \mid \beta} .
\end{aligned}
$$

Let us now introduce the quark fields. Since they are confined on the string, we can write

$$
\psi_{j}=\psi_{j}\left(u^{\alpha}\right),
$$

$j=1,2, \ldots, N$, where $N$ is the number of quark species. (Throughout, repeated indices are summed unless specified otherwise; for the individual quark field, the index is suppressed.) The Dirac matrices, which obey

$$
\left\{\gamma^{\mu}, \gamma^{\nu}\right\}=\gamma^{\mu} \gamma^{\nu}+\gamma^{\nu} \gamma^{\mu}=2 \eta^{\mu \nu},
$$

are chosen to be

$$
\gamma_{i}=\left(\begin{array}{cc}
0 & \sigma_{i} \\
-\sigma_{i} & 0
\end{array}\right), \quad \gamma_{0}=\left(\begin{array}{cc}
0 & -1 \\
-1 & 0
\end{array}\right), \quad \gamma_{5}=\left(\begin{array}{cc}
1 & 0 \\
0 & -1
\end{array}\right),
$$

where $\sigma_{i}$ are the Pauli matrices. $\not a=a_{\mu} \gamma^{\mu}$, for any vector $a_{\mu}$. Now we can write down the action of free quarks confined on a string ${ }^{10}(\hbar=c=1)$,

$$
\begin{aligned}
S & =\int d^{2} u^{\alpha} \sqrt{-g}\left(\frac{1}{2} i \bar{\psi}_{j} \ddot{\phi}_{\|} \psi_{j}-\mathbf{C}\right) \\
& =\int d^{2} u^{\alpha} \mathcal{L},
\end{aligned}
$$

where $\bar{\psi} t^{\alpha} \vec{\partial}_{\beta} \psi=\bar{\psi} t^{\alpha} \partial_{\beta} \psi-\left(\partial_{\beta} \bar{\psi}\right) t^{\alpha} \psi \quad\left(\bar{\psi} \vec{\partial}_{\|} \psi=\bar{\psi} t^{\alpha} \vec{\partial}_{\alpha} \psi\right)$. The constant $\mathfrak{C}$ is the only dimensional parameter of the system and acts as a tension to prevent the system from expanding without check. $d^{2} u^{\alpha} \sqrt{-g}$ is the invariant "volume" element where $g<0$. The tangential derivative $\not_{\|}=\hbar^{\alpha} \partial_{\alpha}$ describes the quark motion along the string. However, each quark field has four components. This means the quark dynamics is not completely specified by the action (2.16) which gives only the tangential motion. To determine the system completely, we resort to the physical picture. Since the string system must be closed, i.e., the conservation of charge and Poincaré generators must be guaranteed, it is required that the normal components of the charge current densities $j_{\mu}$ and the energy-momentum “current" density $\rho_{\mu}^{\alpha}$ must vanish:

$$
\begin{aligned}
& j_{\mu} n^{\mu}=\left(\bar{\psi} \gamma_{\mu} \psi\right) n^{\mu}=j_{\mu} m^{\mu}=0, \\
& \mathcal{P}_{\mu}^{\alpha} n^{\mu}=\mathcal{P}_{\mu}^{\alpha} m^{\mu}=0,
\end{aligned}
$$

where

$$
\begin{aligned}
-\sqrt{-g} p_{\mu}^{\alpha} & =\frac{\delta \mathscr{L}}{\delta \tau_{\alpha}^{\mu}} \\
& =\frac{\delta \sqrt{-g}}{\delta \tau_{\alpha}^{\mu}} \frac{1}{\sqrt{-g}} \mathcal{L}+\sqrt{-g}\left(\frac{1}{2} i \bar{\psi}_{j} \frac{\delta \hbar^{\beta}}{\delta \tau_{\alpha}^{\mu}} \vec{\partial}_{\beta} \psi_{j}\right) \\
& =\tau_{\mu}^{\alpha} \mathcal{L}-\frac{1}{2} i \sqrt{-g} \bar{\psi}_{j}\left[g^{\alpha \beta}\left(\not h n_{\mu}+\not h m_{\mu}\right)+\tau_{\mu}^{\beta} \jmath^{\alpha}\right] \ddot{\partial}_{\beta} \psi_{j} .
\end{aligned}
$$

It is clear that the string part of $\rho_{\mu}^{\alpha}$ is already tangential. Because the quarks are all distinguishable from each other (different quantum numbers) we demand that the normal components of $p_{\mu}^{\alpha}$ of each quark must vanish:

$$
\begin{aligned}
-P_{\mu j}^{\alpha}\left(u^{\beta}\right) & =\frac{1}{2} i g^{\alpha \beta} \bar{\psi}_{j}\left(\not n_{\mu}+\not h m_{\mu}\right) \vec{\partial}_{\beta} \psi_{j} \\
& =0, j \text { not summed }
\end{aligned}
$$

or

$$
\bar{\psi} \overrightarrow{h \partial_{\alpha} \psi}=\bar{\psi} \not h \vec{\partial}_{\alpha} \psi=0 \text {. }
$$

This simply means the momentum of each quark is tangential and Eq. (2.18) may be considered as the normal components of the quark equation of motion.

Although the quarks are confined along the string, they are physical spinors in the $S_{4}$ space; hence "continuity" of each quark field across the string must also be satisfied:

$$
\bar{\psi} \psi=0 \text {. }
$$

Equations (2.17), (2.18), and (2.19) can be considered as constraints on the spinor fields. Together with the action (2.16), the system is completely determined except for boundary conditions. The latter depends on whether the string is open or closed. For an open string, the momentum 
"current" density and the charge current densities must vanish at the ends of the string. For a closed string, all variables must be periodic (antiperiodic for fermions) in the parameter that measures along the length of the string.

The physical picture of the model is clear. The string moves and vibrates while the quarks are moving left and/or right along the string. The distribution and the motion of the quarks affect the shape of the string, which in turn defines the path of the quark motion. Since there is no direct interaction among the quarks, the left-moving and the right-moving quarks pass each other freely. However, the complete solution of the quark fields is not a superposition of the solutions of the leftand right-moving quark fields because of their nonlinear influence on each other via the geometry of the string.

In this work we shall study the string when there is only left-moving or only right-moving quarks. We discover that this system can be solved completely. The complete solution of the subsystem allows one to speculate (a rather natural guess, we believe) at the complete solution of the quarkbinding string we have just formulated.

When there are only left-moving quarks on the string, the boundary condition of the open string at the ends cannot be satisfied, since quarks will flow in and out there. Hence we can only study a closed string; the left-moving quarks become quarks moving clockwise around the string. The first thing we have to do is to project out the clockwise-moving quarks.

We note that from Eq. (2.7),

$$
\not h h=-\not h h,
$$

which provides a signature for the plane $U_{\mu \nu}$. This can be used to specify the direction. The projection operators $\omega_{ \pm}$must satisfy

$$
\psi=\left(\omega_{+}+\omega_{-}\right) \psi=\psi_{+}+\psi_{-}
$$

and

$$
\left(\omega_{ \pm}\right)^{2}=\omega_{ \pm} .
$$

Let us define

$$
\omega_{ \pm}=\frac{1 \pm i \gamma_{5} m \not h}{2}
$$

and study only the case when one projection of the spinor field is present, say

$$
i \gamma_{5} \not h \eta \psi_{+}=\psi_{+} \cdot
$$

That this projects out the left-moving quarks will become clear later. From now on we shall drop the + index whenever it is obvious. The quark species index is implied in Eq. (2.20b) also.

The inclusion of $i \gamma_{5}$ in the choice of $\omega_{+}$in Eq.
(2.20) automatically implies Eqs. (2.19) and (2.17),

$$
\begin{aligned}
\bar{\psi} \psi & =\bar{\psi}\left(i \gamma_{5} m \not h \psi\right) \\
& =\left(i \bar{\psi} \gamma_{5} m \not h\right) \psi \\
& =-\bar{\psi} \psi=0,
\end{aligned}
$$

and

$$
\begin{aligned}
j \cdot n & =\left(\bar{\psi} \gamma_{\mu} \psi\right) n^{\mu}=\bar{\psi} h \psi \\
& =\bar{\psi} h\left(i \gamma_{5} \not h \not \psi\right) \\
& =\left(i \bar{\psi} \gamma_{5} \not h n\right) h \psi \\
& =-\bar{\psi} h \psi=0,
\end{aligned}
$$

and similarly for $j \cdot m=0$. It is useful to note that the normal components of the axial-vector current are also zero,

$$
j_{5 \mu} n^{\mu}=\bar{\psi} h \gamma_{5} \psi=i j_{\mu} m^{\mu}=0 .
$$

Hence, using Eq. (2.9)

$$
j_{5 \mu}=\left(\bar{\psi} x^{\alpha} \gamma_{5} \psi\right) \tau_{\alpha \mu}=J_{5}^{\alpha} \tau_{\alpha \mu} .
$$

That is, both the vector and axial-vector currents are tangential.

Next we demand that the energy-momentum "current" density of the string be tangential also:

$$
\rho_{\mu}^{\beta}=+T^{\beta \alpha} \tau_{\alpha \mu},
$$

where

$$
\begin{aligned}
T^{\alpha \beta} & =\mathfrak{e} g^{\alpha \beta}+\theta^{\alpha \beta} \\
& =e^{\alpha \beta}+\frac{1}{2} i \bar{\psi}_{j} t^{\alpha} \vec{\partial}^{\beta} \psi_{j}-\frac{1}{2} i \bar{\psi}_{j} t^{\delta} \vec{\partial}_{\delta} \psi_{j} g^{\alpha \beta} .
\end{aligned}
$$

This follows from Eq. (2.18), which can be rewritten as, using Eqs. (2.10), (2.11), and (2.21),

$$
\begin{aligned}
\bar{\psi} / \vec{h}_{\alpha} \psi & =-i \partial_{\alpha}\left(\bar{\psi} \not h \gamma_{5} \psi\right)-i \bar{\psi} \gamma_{5}\left(\partial_{\alpha} \not h\right) \psi \\
& =i \nu_{\alpha} \bar{\psi} \gamma_{5} h \psi-i l_{\alpha \beta} \bar{\psi} \gamma_{5} t^{\beta} \psi \\
& =i l_{\alpha \beta} J_{5}^{\beta}=0, \\
\bar{\psi} \not h \vec{\partial}_{\alpha} \psi & =i h_{\alpha \beta} J_{5}^{\beta}=0 .
\end{aligned}
$$

Hence we have a closed system when the axialvector current is identically zero.

It is now straightforward to derive the equations of motion of the system. The Euler-Lagrange equation for the string coordinates is

$$
\begin{aligned}
\partial_{\beta} \frac{\delta \mathscr{L}}{\delta \partial_{\beta} x^{\mu}} & =-\left(\sqrt{-g} T^{\beta \alpha} \tau_{\alpha \mu}\right)_{\mid \beta} \\
& =\sqrt{-g}\left(-T^{\beta \alpha} \tau_{\alpha \mu}\right)_{\| \beta}=0 .
\end{aligned}
$$

The Euler-Lagrange equation of the quark field $\delta \mathcal{L} / \delta \bar{\psi}=\partial_{\alpha} \delta \mathscr{L} / \delta \partial_{\alpha} \bar{\psi}$ becomes

$$
\text { left-hand side }=i \sqrt{-g} t^{\alpha} \partial_{\alpha} \psi
$$

and 


$$
\begin{aligned}
\text { right-hand side } & =-\partial_{\alpha}\left(i \sqrt{-g} t^{\alpha} \psi\right) \\
& =-\frac{1}{2} i \sqrt{-g}\left(t^{\alpha}{ }_{\| \alpha} \psi+t^{\alpha} \partial_{\alpha} \psi\right) .
\end{aligned}
$$

Introducing the mean curvatures

$$
\begin{aligned}
& 2 k_{1}=h_{\alpha}^{\alpha}, \\
& 2 k_{2}=l_{\alpha}^{\alpha}
\end{aligned}
$$

the Dirac equation becomes, using Eq. (2.12),

$$
i \not^{\alpha} \partial_{\alpha} \psi=-i\left(k_{1} \not h+k_{2} \not h\right) \psi \text {. }
$$

It follows that $\theta^{\alpha \beta}=\frac{1}{2} i \bar{\psi}_{j} f^{\alpha} \vec{\partial}^{\beta} \psi_{j}$ since $\bar{\psi} t^{\alpha} \ddot{\partial}_{\alpha} \psi=0$. Projecting out the normal and tangential components of Eq. (2.24) and using Eq. (2.12), we get

$$
\begin{aligned}
& T^{\alpha \beta}{ }_{\| \alpha}=0, \\
& T^{\alpha \beta}=e^{\alpha \beta}+\frac{1}{2} i \bar{\psi}_{j} t^{\alpha} \ddot{\partial}^{\beta} \psi_{j},
\end{aligned}
$$

and

$$
h_{\alpha \beta} T^{\alpha \beta}=l_{\alpha \beta} T^{\alpha \beta}=0,
$$

which are just Eqs. (ii) and (iii) in Table I. Note that these equations of motion can also be derived using the constraints (2.17)-(2.19). Hence they are also the equations of motion for the complete (i.e., with both left- and right-moving quarks) system.

It is straightforward to show that Eq. (2.26) is redundant: One can derive

$$
T_{\| \alpha}^{\alpha \beta}=g^{\beta \gamma}\left(T_{\gamma \| \alpha}^{\alpha}\right)=0
$$

using Eqs. (2.21), (2.23), and (2.25). This means that not all the dynamical variables, namely the quark fields and the string coordinates, are independent. This is a reflection of "gauge invariance" under reparametrization.

To summarize, the system of a closed string, along which massless quarks obeying the projection (2.20) move freely, is completely specified by Eqs. (2.25) and (2.27) together with the periodicity condition on all dynamical variables and the vanishing of the axial-vector current

$$
j_{5 \mu}=\bar{\psi} \gamma_{\mu} \gamma_{5} \psi=0 \text {. }
$$

Classically, any system involving fermions is plagued with negative energies. In particular the fermion energy density is not positive-definite. We shall ignore this difficulty by assuming that the fermion energy density is positive-definite, which is the case after proper fermion-antifermion identification and Fermi statistics are taken into account. This is discussed in Sec. IV.

In the absence of quark fields, the quark-binding string action reduces to the Nambu or the empty string action. This is clear from the original action (2.16) and also from the final spectrum, as we shall see later.

\section{SOLUTION}

The scheme of solving the set of coupled nonlinear equations of motion (2.25) and (2.27) is as follows: First we derive some properties of the system, which are essential for the solution. We then solve the geometry of the closed string in terms of the fermion distribution by adopting a particular coordinate choice. Finally we go back to solve the Dirac equations in terms of the geometry. Supplementary discussions and details are relegated to Appendixes $\mathrm{A}, \mathrm{B}$, and $\mathrm{C}$.

\section{A. Properties of the system}

Here we derive some of the properties of the system. In particular, we show that there exists a parameter choice such that the string coordinates obey the free-string equation of motion. For convenience we write

$$
g_{\alpha \beta}=\left(\begin{array}{cc}
A & V \\
V & B
\end{array}\right),
$$

so that $g=A B-V^{2}<0$, since the signature of the metric is $(1,-1)$. Let us also introduce, for any function $W\left(u^{\alpha}\right)$, the following notation:

$$
\dot{W}=\frac{\partial W}{\partial u^{0}}, \quad W^{\prime}=\frac{\partial W}{\partial u^{1}} .
$$

Using Eqs. (2.4) and (2.8), the projection (2.20) can be expressed in terms of $\tau_{\alpha \mu}$,

$$
i \gamma_{5} \not h i h=\frac{V-\not_{0} t_{1}}{\sqrt{-g}} \text {. }
$$

Next we observe that the current is a null vector; in fact (see Appendix A)

$$
j_{i} \psi_{k}=\left(\bar{\psi}_{i} \gamma_{\mu} \psi_{i}\right) \gamma^{\mu} \psi_{k}=0, \quad i \text { not summed }
$$

for both $i=k$ and $i \neq k$. Multiplying $\bar{\psi}_{k}$ on the left, we get

$$
j_{i \mu} j_{k}^{\mu}=J_{i \alpha} J_{k}^{\alpha}=0 \text {. }
$$

Also

$$
J_{\alpha} \theta^{\alpha \beta}=0,
$$

where $\theta^{\alpha \beta}$ is the fermion part of the energy-momentum tensor (2.22), or

$$
J_{\alpha} T^{\alpha \beta}=\mathcal{e} J^{\beta} \text {. }
$$

Using the Dirac equation (2.25), we also obtain

$$
\begin{gathered}
J^{\alpha}{ }_{\| \alpha}=0, \\
T_{\alpha}^{\alpha}=2 e .
\end{gathered}
$$

To study the string geometry, we can, for the moment, concentrate only on Eqs. (2.26), (2.27), $(3.4),(3.5)$, and (3.6). The quarks appear only in 
the form of the current and their energy-momentum density $\theta^{\alpha \beta}$.

The original action is independent of the choice of the parameters $u_{\alpha}$. Since $T^{\alpha \beta}$ is symmetric and has signature $(1,-1)$ (see Appendix B), we choose $T^{\alpha \beta}$ to be off-diagonal:

$$
T^{\alpha \beta}=\mathcal{C} T^{01}\left(\begin{array}{ll}
0 & 1 \\
1 & 0
\end{array}\right) .
$$

That this is possible should be clear: Giren $T^{\alpha \beta}\left(u^{0}, u^{1}\right)$, a local coordinate system can be found, $\left(u^{0}, u^{1}\right) \rightarrow(\tau, \sigma)$, such that $T^{\alpha \beta}(\tau, \sigma)$ is offdiagonal.

Using Eqs. (3.1) and (3.6), $T^{\alpha \beta}$ becomes

$$
T^{\alpha \beta}=\frac{\mathfrak{e}}{V}\left(\begin{array}{ll}
0 & 1 \\
1 & 0
\end{array}\right) .
$$

As we shall see, $V=\dot{x} \cdot x^{\prime}$ must be nonzero. Using Eq. (3.5)

$$
\mathcal{C} J_{\beta} J^{\beta}=J_{\alpha} T^{\alpha \beta} J_{\beta}=\frac{2 \mathfrak{C} J_{0} J_{1}}{V}=0 .
$$

We find that either $J_{0}$ or $J_{1}$ must be zero. We choose (this defines our sign convention)

$$
J_{1}=0 \text {. }
$$

Then from Eq. (3.4),

$$
J_{\alpha} J^{\alpha} \equiv g^{\alpha \beta} J_{\alpha} J_{\beta}=0,
$$

we obtain $g^{00}=0$, which means

$$
g_{\alpha \beta}=\left(\begin{array}{cc}
A & V \\
V & 0
\end{array}\right)
$$

and

$$
g^{\alpha \beta}=\frac{1}{V}\left(\begin{array}{cc}
0 & 1 \\
1 & -A / V
\end{array}\right),
$$

so that $-g=V^{2}>0$. This gives

$$
\begin{aligned}
\theta_{\alpha \beta} & =\mathfrak{e}\left(\begin{array}{ll}
A & 0 \\
0 & 0
\end{array}\right) \\
& =\frac{1}{2} i \bar{\psi}_{j} t_{\alpha} \vec{\partial}_{\beta} \psi_{j} .
\end{aligned}
$$

It should be clear that we cannot choose $J_{i_{1}}=0$ for some quarks while $J_{k 0}=0$ for some other quarks.

The relation between the coordinates and the quark fields is manifest:

$$
\mathfrak{e} A=\mathfrak{e} \dot{x}^{2}=\frac{1}{2} i \bar{\psi}_{j} t_{0} \vec{\partial}_{0} \psi_{j} \cdot
$$

The Christoffel symbols of the second kind now simplify to

$$
\begin{aligned}
& \left\{\begin{array}{c}
0 \\
0
\end{array}\right\}=(\ln V)^{\circ}-A^{\prime} / 2 V, \\
& \left\{\begin{array}{c}
1 \\
1
\end{array}\right\}=(\ln V)^{\prime},
\end{aligned}
$$

$$
\begin{aligned}
& \left\{\begin{array}{c}
1 \\
0
\end{array}\right\}=A^{\prime} / 2 V, \\
& \left\{\begin{array}{cc}
1 \\
0 & 0
\end{array}\right\}=\frac{\dot{A}}{2 V}-\frac{A}{V^{2}}\left(\dot{V}-A^{\prime} / 2\right), \\
& \left\{\begin{array}{cc}
0 \\
1
\end{array}\right\}=\left\{\begin{array}{cc}
0 \\
0 & 1
\end{array}\right\}=0 .
\end{aligned}
$$

Using these and Eq. (2.26)

$$
T^{\alpha 1}{ }_{\| \alpha}=0 \text {, }
$$

we obtain $A^{\prime}=0$, so that the Christoffel symbols are further simplified:

$$
\begin{aligned}
& \left\{\begin{array}{cc}
0 & 0 \\
0 & 0
\end{array}\right\}=(\ln V)^{\circ}, \\
& \left\{\begin{array}{cc}
1 & 1 \\
1 & 1
\end{array}\right\}=(\ln V)^{\prime}, \\
& \left\{\begin{array}{cc}
1 & 0 \\
0 & 0
\end{array}\right\}=\frac{\dot{A}}{2 V}-\frac{A \dot{V}}{V^{2}}, \\
& \left\{\begin{array}{cc}
0 \\
0 & 1
\end{array}\right\}=\left\{\begin{array}{cc}
0 & 1 \\
1 & 1
\end{array}\right\}=\left\{\begin{array}{cc}
1 & 1 \\
0 & 1
\end{array}\right\}=0 .
\end{aligned}
$$

Equation (2.27) immediately gives

$$
h_{01}=l_{01}=0 \text {. }
$$

Equation (2.12) becomes, when written explicitly,

$$
\ddot{x}_{\mu}=\dot{x}_{\mu}(\ln V)^{\bullet}+h_{00} n_{\mu}+l_{00} m_{\mu}+\left(\frac{\dot{A}}{2 V}-\frac{A \dot{V}}{V^{2}}\right) x_{\mu}^{\prime},
$$

$$
\begin{aligned}
& x_{\mu}^{\prime \prime}=x_{\mu}^{\prime}(\ln V)^{\prime}+h_{11} n_{\mu}+l_{11} m_{\mu}, \\
& \dot{x}_{\mu}^{\prime}=0,
\end{aligned}
$$

the last of which allows us to write

$$
x_{\mu}(\tau, \sigma)=S_{\mu}(\sigma)+Q_{\mu}(\tau) .
$$

\section{B. The geometry}

The embedding of the string in Minkowski space requires the introduction of the metric and the curvature tensors. The consistency of the geometry is guaranteed by the integrability conditions, namely, the Gauss-Codazzi equations ${ }^{9}$

$$
\begin{aligned}
& R_{\alpha \beta \gamma \delta}=\left(h_{\alpha \gamma} h_{\beta \delta}-h_{\alpha \beta} h_{\gamma \delta}\right)+\left(l_{\alpha \gamma} l_{\beta \delta}-l_{\alpha \beta} l_{\gamma \delta}\right), \\
& h_{\alpha \beta \| \gamma}-h_{\alpha \gamma \| \beta}=\nu_{\gamma} l_{\alpha \beta}-\nu_{\beta} l_{\alpha \gamma}, \\
& l_{\alpha \beta \| \gamma}-l_{\alpha \gamma \| \beta}=-\nu_{\gamma} h_{\alpha \beta}+\nu_{\beta} h_{\alpha \gamma},
\end{aligned}
$$

where the Riemann curvature tensor is given by

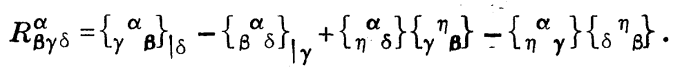

When the curvature tensors are all constructed from the metric tensor, the Gauss-Codazzi equations are automatically satisfied. However, since we are going to solve the string by making a coordinate choice which apparently breaks Lorentz covariance, it is reassuring to check that the integrability constraints are satisfied.

From its symmetry properties, it is clear that 
the Riemann tensor has only one independent component. Using Eq. (3.9) the Gauss-Codazzi equations become

$$
\begin{aligned}
& h_{00}^{\prime}+\frac{1}{V}\left(\frac{\dot{A}}{2}-\frac{A \dot{V}}{V}\right) h_{11}=\nu_{1} l_{00}, \\
& l_{00}^{\prime}+\frac{1}{V}\left(\frac{\dot{A}}{2}-\frac{A \dot{V}}{V}\right) l_{11}=-\nu_{1} h_{00}, \\
& \dot{h}_{11}=\nu_{0} l_{11}, \\
& \nu_{0} h_{11}=-\dot{i}_{11} .
\end{aligned}
$$

We observe that the theory has conformal invariance; that is, Eqs. (3.10), (3.11), (3.12), (3.14), and (3.15) are form-invariant under the conformal transformation

$$
\begin{gathered}
\tau \rightarrow e(\tau), \\
\sigma \rightarrow f(\sigma) .
\end{gathered}
$$

Hence we can choose from Eq. (3.13)

$$
x_{+}=p(\tau+\sigma)=x_{0}+x_{3},
$$

where $p$ is a constant. Then the constraint among the string coordinates

$$
g_{11} \equiv x^{\prime 2}=0
$$

can be written as

$$
x_{-}^{\prime}=\frac{1}{p}\left(x_{1}^{\prime 2}+x_{2}^{\prime 2}\right),
$$

and Eq. (3.8) becomes

$$
\dot{x}_{-}=\frac{1}{p}\left(\dot{x}_{1}{ }^{2}+\dot{x}_{2}{ }^{2}+A\right) \text {. }
$$

Hence $x_{1}, x_{2}$, and $A$ can be taken to be the independent dynamical variables, with

$$
2 V=\left(\dot{x}_{1}-\dot{x}_{2}\right)^{2}+\left(x_{1}^{\prime}-x_{2}^{\prime}\right)^{2}+A \text {. }
$$

Similarly $h_{\alpha \beta}$ and $l_{\alpha \beta}$ can be expressed in terms of $x_{1}, x_{2}$, and $A$. The Gauss-Codazzi equations can then be explicitly verified (see Appendix C).

Actually there is another Gauss-Codazzi equation:

$$
\left.\nu_{\beta}\right|_{\alpha}-\nu_{\left.\alpha\right|_{B}}+l_{\alpha \gamma} h_{\beta}^{\gamma}-h_{\beta \gamma} l_{\alpha}^{\gamma}=0 .
$$

Since all physical quantities are tangential, this is automatically satisfied.

\section{The Dirac equation}

Equation (3.2) becomes

$$
\begin{aligned}
& t_{0} t_{1}=V\left(1-i \gamma_{5} \not h n\right), \\
& t_{1} t_{0}=V\left(1+i \gamma_{5} \not h n\right) .
\end{aligned}
$$

or

Therefore, with $A=\not_{0} t_{0}$,

$$
t_{1} \psi=\frac{1}{A} t_{0}\left(t_{0} t_{1}\right) \psi=0 .
$$

Differentiating with respect to $\tau$, we obtain, using Eq. (3.13),

$$
\partial_{0}\left(t_{1} \psi\right)=t_{1} \dot{\psi}=0 \text {. }
$$

Differentiating with respect to $\sigma$, we obtain, using Eq. (3.12),

$$
t_{1} \psi^{\prime}=-\left(h_{11} \not h+l_{11} \not h\right) \psi \text {. }
$$

Note that

$$
\begin{aligned}
& k_{1}=\frac{1}{2} h^{\alpha}{ }_{\alpha}=-\frac{A}{2 V^{2}} h_{11}, \\
& k_{2}=-\frac{A}{2 V^{2}} l_{11} .
\end{aligned}
$$

Substituting the above four equations into the Dirac equation (3.25), we obtain

$$
g^{01} \hbar_{0} \psi^{\prime}=-\frac{A}{2 V^{2}}\left(h_{11} \not h+l_{11} \not h\right) \psi \text {. }
$$

Multiplying this on the left by $t_{0}$ and using Eqs. (3.11) and (3.19), the Dirac equation becomes

$$
\psi^{\prime}=-\frac{t_{0} t_{1}^{\prime}}{2 V} \psi
$$

Equation (3.19) puts a constraint among the components of $\psi$. Using this to eliminate the dependent components, we can write $\psi$ in terms of the two unknown functions $u, v$,

$$
\psi=\left[\begin{array}{c}
u \\
x_{r}^{\prime} u / p \\
-x_{l}^{\prime} v / p \\
v
\end{array}\right]
$$

where

$$
x_{r}^{\prime}=x_{l}^{\prime *}=x_{1}^{\prime}+i x_{2}^{\prime} .
$$

The Dirac equation can now be written in terms of $u, v$ :

$$
\begin{aligned}
& u^{\prime}=-\frac{\left(x_{l}^{\prime}-\dot{x}_{l}\right) x_{r}^{\prime \prime}}{2 V} u, \\
& v^{\prime}=-\frac{\left(x_{r}^{\prime}-\dot{x}_{r}\right) x_{l}^{\prime \prime}}{2 V} v .
\end{aligned}
$$

Solving this, we obtain

$$
\psi=\left[\begin{array}{c}
1 \\
x_{r}^{\prime} / p \\
0 \\
0
\end{array}\right] L F(\tau)+\left[\begin{array}{c}
0 \\
0 \\
-x_{l}^{\prime} / p \\
1
\end{array}\right] R G(\tau),
$$

where 


$$
L=R^{\dagger}=\exp \left[-\frac{1}{2} \int^{\sigma} \frac{\left(x_{l}^{\prime}-\dot{x}_{l}\right) x_{r}^{\prime \prime}}{V} d \sigma_{1}\right]
$$

and $F(\tau)$ and $G(\tau)$ are arbitrary functions of $\tau$. This is an implicit solution of $\psi$ since $L$ is a function of $A$ (via $V$ ) which in turn is a function of the quark fields [see Eqs. (3.8) and (3.18)].

Since the axial-vector current is tangential, the only nontrivial component of $j_{5 \mu}$ in Eq. (2.28) is, using Eq. (3.19),

$$
\bar{\psi} t_{0} \gamma_{5} \psi=\frac{2}{p}\left(G^{\dagger} G-F^{\dagger} F\right)=0 .
$$

This means $F$ and $G$ differ by a phase only; we choose $F=G$. It is clear that if $\psi$ is a solution of the system, so is

$$
\psi_{c}=C \psi^{*}=\left(i \gamma_{2}\right) \psi^{*},
$$

where $C \gamma^{0}$ is the charge-conjugation matrix. Explicitly, we have

$$
i \gamma_{5} \not h h \psi_{c}=\psi_{c}
$$

and

$$
i f^{\alpha} \partial_{\alpha} \psi_{c}=-i\left(k_{1} \not h+k_{2} \not h\right) \psi_{c} .
$$

Here

$$
\begin{aligned}
\psi_{c} & =i \gamma_{2}\left[\begin{array}{c}
L F \\
\left(x_{r}^{\prime} / p\right) L F \\
-\left(x_{l}^{\prime} / p\right) R F \\
R F
\end{array}\right] * \\
& =\left[\begin{array}{c}
L \\
\left(x_{r}^{\prime} / p\right) L \\
-\left(x_{l}^{\prime} / p\right) R \\
R
\end{array}\right] F^{*} .
\end{aligned}
$$

This means $\psi$ should have both quark and antiquark solutions. Using its antiperiodic property (with the period $\tau_{0}$ taken to be 1) $F(\tau)$ can be expanded into Fourier components. Hence

$$
\psi=\Re\left[\begin{array}{c}
L \\
\left(x_{r}^{\prime} / p\right) L \\
-\left(x_{l}^{\prime} / p\right) R \\
R
\end{array}\right] \sum_{m=-\infty}^{\infty} b_{m} e^{-2 \pi i m \tau},
$$

where the prime indicates the sum over half-integers with coefficients $b_{m}$, and $\Re$ is the normalization to be determined. A Dirac field has fermion and antifermion solutions, each with two spin polarizations. The projection (2.20) is a geometric one, leaving one solution each for the fermion and the antifermion. The confinement on a twodimensional manifold has deprived the Dirac particles of their conventional spin structures. The reader probably would have guessed this from the fact that the energy-momentum tensor $T^{\alpha \beta}$ is symmetric without the introduction of any "superpotential" terms. We should also expect the absence of the spin part of the angular momentum tensor density $M^{\alpha \mu \nu}$, which is given by

$$
\begin{aligned}
M^{\alpha \mu \nu}= & x^{\mu} \rho^{\alpha \nu}-x^{\nu} \rho^{\alpha \mu} \\
& +\frac{1}{4} \bar{\psi}_{j}\left(f^{\alpha} \sigma^{\mu \nu}+\sigma^{\mu \nu} f^{\alpha}\right) \psi_{j},
\end{aligned}
$$

where

$$
\sigma^{\mu \nu}=\frac{1}{2} i\left[\gamma^{\mu}, \gamma^{\nu}\right]
$$

It is straightforward to verify this is indeed true, that is,

$$
\frac{1}{2} \bar{\psi}\left(\boldsymbol{t}^{\alpha} \sigma^{\mu \nu}+\sigma^{\mu \nu} \mathfrak{f}^{\alpha}\right) \psi=0
$$

using Eqs. (3.20) and (3.21). Note that

$$
L R=L L^{\dagger}=1 / V .
$$

Using this, we see that $\mathrm{e} A$ is quite simple despite the complicated form of $\psi$

$$
\begin{aligned}
\mathbf{e} A & =\frac{1}{2} i \bar{\psi}_{j} t_{0} \vec{\partial}_{0} \psi_{j} \\
& =\frac{2 i}{p}\left(F_{j}^{\dagger} \dot{F}_{j}-\stackrel{\circ}{F}_{j}^{\dagger} F_{j}\right) .
\end{aligned}
$$

Poincare covariance should be satisfied, since the original formulation is Lorentz-covariant.

\section{THE QUANTIZED SYSTEM}

\section{A. Noncovariant quantization}

We choose our units such that $\mathfrak{e}=1$. We shall restore it only at the end. The energy-momentum is given by

$$
P_{\mu}=\int d \Sigma_{\alpha} \sqrt{-g} \rho_{\mu}^{\alpha},
$$

where the integral is to be taken at any fixed time $t$

$$
t=\tau+\sigma .
$$

This means the integration is over any spacelike submanifold, where the spacelike parameter

$$
\rho=\frac{1}{2}(\tau-\sigma)
$$

actually measures along the string. To see this, we observe that $P_{-}$is the generator of infinitesimal translations in the $x_{+}$direction [see Eq. (3.16)]; therefore, the Hamiltonian should be given by

$$
H=P_{+} P_{-} .
$$

To evaluate $H$, let us expand $x^{\mu}$ as follows, which is permitted by Eq. (3.13): 


$$
\begin{aligned}
x^{\mu}(\tau, \sigma)= & \frac{1}{2 \sqrt{\pi}} q^{\mu}+\sqrt{\pi} a_{0}^{\mu} \tau+\sqrt{\pi} c_{0}^{\mu} \sigma \\
& +\frac{i}{2 \sqrt{\pi}} \sum_{n \neq 0}\left(\frac{a_{n}^{\mu}}{n} e^{-2 \pi i n \tau}+\frac{c_{n}^{\mu}}{n} e^{-2 \pi i n \sigma}\right),
\end{aligned}
$$

where the period of $\sigma$ is also taken to be unity. For fixed $t$, the periodicity condition of $x^{\mu}$ is

$$
x^{\mu}(t, \rho)=x^{\mu}(t, \rho+1)
$$

This implies $a_{0}^{\mu}=c_{0}^{\mu}$. Reality of $x^{\mu}$ implies

$$
q_{\mu}=q_{\mu}^{*}, \quad a_{n}^{\dagger}=a_{-n}, \quad a_{0}=a_{0}^{*}, \quad c_{n}^{\dagger}=c_{-n} .
$$

Equation (3.16) then gives

$$
\begin{aligned}
& q_{+}=a_{n}^{+}=c_{n}^{+}=0, \quad n \neq 0 \\
& p=\sqrt{\pi} a_{0}^{+} .
\end{aligned}
$$

Therefore, from Eqs. (3.17) and (4.5) we obtain

$$
\begin{aligned}
c_{n}^{-} & =\frac{2 \tilde{\mathscr{L}}_{n}}{a_{0}^{+}}, \\
\tilde{\mathscr{L}}_{n} & =\frac{1}{2} \sum_{l=-\infty}^{\infty} c_{-l} \cdot c_{n+l} \\
& =\frac{1}{2} \sum_{l}\left(c_{-l}^{1} c_{n+l}^{1}+c_{-l}^{2} c_{n+l}^{2}\right) .
\end{aligned}
$$

To calculate the dependent modes $a_{n}^{-}$, we have to fix the normalization of the fermion fields. Consider the conserved charge $Q$,

$$
\begin{aligned}
Q & =\int d \Sigma_{\alpha} \sqrt{-g} J^{\alpha} \\
& =\int d \Sigma_{\alpha} \sqrt{-g}\left(\bar{\psi} t^{\alpha} \psi\right) \\
& =\frac{4}{p} \int_{0}^{1} d \tau F^{\dagger} F \\
& =\Re^{2} \frac{4}{p} \sum^{\prime} b_{m}^{\dagger} b_{m} .
\end{aligned}
$$

This suggests the normalization $\Re^{2}=p / 4$ to be a convenient one. Now, we can put Eqs. (3.18), $(3.23),(4.5),(4.6)$, and (4.8) together to obtain

$$
\begin{aligned}
& a_{n}^{-}=\frac{2 \mathcal{L}_{n}}{a_{0}^{+}} \\
& \mathscr{L}_{n}=\frac{1}{2} \sum_{l} a_{-l} \cdot a_{n+l}+\sum_{m}^{\prime}\left(m+\frac{1}{2} n\right) b_{j m}^{+} b_{j, m+n}
\end{aligned}
$$

and

$$
P_{-}=\frac{2 \sqrt{\pi}}{a_{0}^{+}}\left(\mathcal{L}_{0}+\tilde{L}_{0}\right),
$$

where periodicity requires $c_{0}^{-}=a_{0}^{-}$, or

$$
\mathscr{L}_{0}=\tilde{\mathscr{L}}_{0} .
$$

The Hamiltonian is now given by

$$
\begin{aligned}
H= & 4 \pi\left(\mathcal{L}_{0}+\tilde{\mathscr{L}}_{0}\right) \\
=4 \pi & {\left[\sum_{n=1}^{\infty}\left(a_{n}^{\dagger} \cdot a_{n}+c_{n}^{\dagger} \cdot c_{n}\right)+a_{0} \cdot a_{0}\right.} \\
& \left.+\sum_{m}^{\prime} m b_{j m}^{\dagger} b_{j m}\right],
\end{aligned}
$$

where all the independent modes appear in bilinear forms. Furthermore, $H$ is already diagonalized. This suggests that, if we introduce canonical quantization to these modes, any function which can be expressed in these modes automatically satisfies the Hamiltonian equation of motion. We shall not burden the reader with details. The quantized system is specified as follows:

$$
\begin{aligned}
& d_{m}=b_{-m}^{\dagger}, \quad m>0 \\
& d_{m}^{\dagger}=b_{-m}
\end{aligned}
$$

are respectively the annihilation and creation operators for antiquark modes while $b_{m}, b_{m}^{\dagger}(m>0)$ are those for the quark modes. They obey

$$
\left\{b_{i n}, b_{j m}^{\dagger}\right\}=\left\{d_{i n}, d_{j m}^{\dagger}\right\}=\delta_{m, n} \delta_{i j},
$$

where $i$ and $j$ are quark species indices. We also have

$$
\begin{aligned}
& {\left[a_{n}^{i}, a_{l}^{j}\right]=n \delta_{n,-l} \delta^{i j},} \\
& {\left[c_{n}^{i}, c_{l}^{j}\right]=n \delta_{n,-l} \delta^{i j},} \\
& {\left[q^{i}, a_{0}^{j}\right]=i \delta^{i j},} \\
& {\left[q_{-}, a_{0}^{+}\right]=-i,}
\end{aligned}
$$

where the upper indices $i, j=1,2$ are the spacetime indices. All other commutators (or anticommutators) among $b_{m}, d_{m}, c_{n}, a_{n}, q^{i}, q_{-}, a_{0}^{j}$, and $a_{0}^{+}$are zero. Ambiguities arising from the ordering of operators are resolved by normal-ordering with respect to the ground state $|0\rangle$

$$
\begin{aligned}
& b_{m}|0\rangle=d_{m}|0\rangle, \quad m>0 \\
& a_{n}^{i}|0\rangle=c_{n}^{i}|0\rangle, \quad n>0 .
\end{aligned}
$$

Hence the charge operator becomes

$$
Q=\sum_{m>0}^{\prime}\left(b_{m}^{\dagger} b_{m}-d_{m}^{\dagger} d_{m}\right)+Q_{0}
$$


Also

$$
\begin{aligned}
& \tilde{\mathscr{L}}_{0}=\sum_{n=1}^{\infty} c_{n}^{\dagger} \cdot c_{n}+\frac{a_{0}^{2}}{2}, \\
& \mathscr{L}_{0}=\sum_{n=1}^{\infty} a_{n}^{\dagger} \cdot a_{n}+\frac{a_{0}^{2}}{2}+\sum_{m>0}^{\prime} m\left(b_{j m}^{\dagger} b_{j m}+d_{j m}^{\dagger} d_{j m}\right),
\end{aligned}
$$

where

$$
\begin{aligned}
& c_{0}^{-} a_{0}^{+}=\tilde{\mathscr{L}}_{0}-\tilde{\alpha}_{0}, \\
& a_{0}^{-} a_{0}^{+}=\mathscr{L}_{0}-\alpha_{0} .
\end{aligned}
$$

$\alpha_{0}, \tilde{\alpha}_{0}$, and $Q_{0}$ are constants arising from normalordering. Since the charge of the empty string is zero, we shall assume $Q_{0}=0$. Condition (4.10) can be imposed weakly, i.e., all physical states $|\Omega\rangle$ must satisfy

$$
\left(\mathscr{L}_{0}-\alpha_{0}\right)|\Omega\rangle=\left(\tilde{\mathscr{L}}_{0}-\tilde{\alpha}_{0}\right)|\Omega\rangle .
$$

Since $a_{0}^{\mu}$ is the momentum of the string, the mass $\left(\Re^{2}\right)$ spectrum follows immediately from the Hamiltonian,

$$
\mathfrak{T l}^{2}=4 \pi \mathfrak{C}\left(\mathscr{L}_{0}+\tilde{\mathscr{L}}_{0}-a_{0}^{2}-\alpha_{0}-\tilde{\alpha}_{0}\right),
$$

where we restore the dimensional parameter $\boldsymbol{e}$. The Regge trajectories are linear with slope $\alpha^{\prime}$ $=1 / 8 \pi \mathrm{e}$ and intercept $\left(\alpha_{0}+\tilde{\alpha}_{0}\right)$.

Some useful commutators are given in Appendix D.

\section{B. No Lorentz covariance}

Since the quantization is noncovariant, Lorentz covariance has to be examined. To construct the angular momentum operators, the ordering problem arises again. This can be resolved by symmetrizing the classical expression in $x_{\mu}$ and $\rho_{\nu}^{\alpha}$ to ensure that $M^{\mu \nu}$ is a Hermitian operator

$$
\begin{aligned}
M^{\mu \nu}= & \int d \Sigma_{\alpha} M^{\alpha \mu \nu} \sqrt{-g} \\
=\frac{1}{2} \int d \Sigma_{\alpha} \sqrt{-g}\left(x^{\mu} \rho^{\alpha \nu}-x^{\nu} \rho^{\alpha \mu}\right. & \\
& \left.+\rho^{\alpha \nu} x^{\mu}-\rho^{\alpha \mu} x^{\nu}\right),
\end{aligned}
$$

since we have shown earlier that the spin part of $M^{\alpha \mu \nu}$ is zero. The angular momentum operators are

$$
\begin{aligned}
M^{12}=q^{1} a_{0}^{2}-q^{2} a_{0}^{1}-i \sum_{n=1}^{\infty} \frac{1}{n}\left(a_{-n}^{1} a_{n}^{2}-a_{-n}^{2} a_{n}^{1}\right. \\
\left.+c_{-n}^{1} c_{n}^{2}-c_{-n}^{2} c_{n}^{1}\right),
\end{aligned}
$$

$$
\begin{aligned}
& M^{i+}=q^{i} a_{0}^{+}, \\
& M^{+-}=-\frac{1}{2}\left(q^{-} a_{0}^{+}+a_{0}^{+} q^{-}\right)-4 \pi E \rho, \\
& M^{i-}=M_{+}^{i-}+M_{-}^{i-}-q^{-} a_{0}^{i}-\frac{2 \pi}{a_{0}^{+}}\left(a_{0}^{i} E+E a_{0}^{i}\right) \rho,
\end{aligned}
$$

where

$$
\begin{aligned}
M_{+}^{i-}= & \frac{1}{2}\left[q^{i} \frac{1}{a_{0}^{+}}\left(\mathscr{L}_{0}-\alpha_{0}\right)+\frac{1}{a_{0}^{+}}\left(\mathscr{L}_{0}-\alpha_{0}\right) q^{i}\right] \\
& -\frac{i}{a_{0}^{+}} \sum_{n=i}^{\infty} \frac{a_{-n}^{i} \mathscr{L}_{n}-\mathcal{L}_{-n} a_{n}^{i},}{n} \\
M_{-}^{i-}= & \frac{1}{2}\left[q^{i} \frac{1}{a_{0}^{+}}\left(\tilde{\mathscr{L}}_{0}-\tilde{\alpha}_{0}\right)+\frac{1}{a_{0}^{+}}\left(\tilde{\mathscr{L}}_{0}-\tilde{\alpha}_{0}\right) q^{i}\right] \\
& -\frac{i}{a_{0}^{+}} \sum_{n=1}^{\infty} \frac{c_{-n}^{i} \tilde{\mathscr{L}}_{n}-\tilde{\mathscr{L}}_{-n} c_{n}^{i}}{n}, \\
E= & \left(\mathcal{L}_{0}-\alpha_{0}\right)-\left(\tilde{\mathscr{L}}_{0}-\tilde{\alpha}_{0}\right) .
\end{aligned}
$$

The presence of an explicit dependence on $\rho$ does not violate the conservation of angular momentum since we have $E|\Omega\rangle=0$ and $E$ commutes with all $M^{\mu \nu}$ and the Hamiltonian. It is straightforward to verify that all commutation relations of $M^{\mu \nu}$ are satisfied except $\left[\mathrm{M}^{1-}, \mathrm{M}^{2-}\right]$, which must be zero for the Lorentz algebra to close. (Appendix D contains some useful commutators.) Define

$$
\begin{aligned}
& \mathscr{L}_{n}^{\prime}=\mathscr{L}_{n}-a_{0} \cdot a_{n}, \\
& \tilde{\mathscr{L}}_{n}^{\prime}=\tilde{\mathcal{L}}_{n}-a_{0} \cdot c_{n} .
\end{aligned}
$$

The troublesome part of $M^{i-}$ can be subdivided into the left-moving part

$$
M_{L}^{i-}=-\frac{i}{a_{0}^{+}} \sum_{n=1}^{\infty} \frac{a_{-n}^{i} \mathcal{L}_{n}^{\prime}-\mathcal{L}_{-n}^{\prime} a_{n}^{i}}{n}
$$

and the right-moving part

$$
M_{R}^{i-}=-\frac{i}{a_{0}^{+}} \sum_{n=1}^{\infty} \frac{c_{-n}^{i} \tilde{\mathcal{L}}_{n}^{\prime}-\tilde{\mathscr{L}}_{-n}^{\prime} c_{n}^{i}}{n} .
$$

It is straightforward (though tedious) to verify

$$
\begin{aligned}
a_{0}^{+2}\left[M_{L}^{i-}, M_{L}^{j-}\right]= & 2\left(\mathcal{L}_{0}^{\prime}-\frac{N+2}{24}\right) \sum_{n=1}^{\infty} \frac{a_{-n}^{i} a_{n}^{j}-a_{-n}^{j} a_{n}^{i}}{n} \\
& +\left(1-\frac{N+2}{24}\right) \sum_{n=1}^{\infty} n\left(a_{-n}^{i} a_{n}^{j}-a_{-n}^{j} a_{n}^{i}\right),
\end{aligned}
$$

$a_{0}^{+2}\left[M_{L}^{i-}, M_{R}^{i-}\right]=0$,

and

$$
\begin{aligned}
a_{0}^{+2}\left[M_{R}^{i-}, M_{R}^{j-}\right]= & 2\left(\tilde{\mathscr{L}}_{0}^{\prime}-\frac{N^{\prime}+2}{24}\right) \sum_{n=1}^{\infty} \frac{c_{-n}^{i} c_{n}^{j}-c_{-n}^{j} c_{n}^{i}}{n} \\
& +\left(1-\frac{N^{\prime}+2}{24}\right) \sum_{n=1}^{\infty} n\left(c_{-n}^{i} c_{n}^{j}-c_{-n}^{j} c_{n}^{i}\right)
\end{aligned}
$$


where $N$ and $N^{\prime}$ are the number of quarks moving left and right, respectively. For the subsystem we have been considering so far, $N^{\prime}=0$. Hence the last term in Eq. (4.23) does not vanish in the commutator $\left[\mathrm{M}^{1-}, \mathrm{M}^{2-}\right]$ and the Lorentz algebra is not closed. However, it is still interesting to note that the commutator of the left-moving part of $\left[M^{1-}, M^{2-}\right.$ ] vanishes if $N=22$ and $\alpha_{0}=1$ [where Eq. (4.22) is used]. If, at the beginning, we choose

$$
-i \gamma_{5} \not h h \psi_{j}=\psi_{j}, \quad j=1,2, \ldots, N^{\prime}
$$

instead of the projection (2.20), then the left-moving part of $\left[\mathrm{M}^{1-}, M^{2-}\right]$ will not vanish while the rightmoving part vanishes if $N^{\prime}=22$ and $\tilde{\alpha}_{0}=1$. The inclusion of $N_{s}$ number of massless scalar fields moving in the same direction as the fermion fields (say, left-moving) changes the condition on the number of fermions to ${ }^{11}$

$$
N \leqslant N+N_{s}=22 \text {. }
$$

That this nonlinear representation of the Lorentz algebra does not close is due to the normal-ordering of the operators. This is purely a quantummechanical effect.

\section{DISCUSSIONS AND SPECULATIONS}

\section{A. The quark-binding string}

We have shown that the well-defined, covariant subsystem of quarks moving clockwise around a closed string does not have a relativistic quantummechanical solution. Mathematically it is due to the nonlinear dependence among the dynamical variables. We are very much aware that there may exist singular modes in the solution which we have discarded by fiat; their presence may restore the much wanted Lorentz covariance. Also it is possible that our quantization procedure is incorrect; to resolve the difficulty, we just have to find the proper quantization. In either case, it may require slight modifications in the original formulation of the string. Barring these possibilities, we believe that quantum mechanics and relativity put very strong constraints on the nonlinear system. To interpret boldly the negative results we are facing, we shall go into a wide spree of speculations. No apology is offered to the cautious readers, since the unsupported statements to be made are so precise that we are confident they can be proved or disproved in the very near future.

We shall formulate our speculations in the form of a conjecture. First we make a few observations, keeping in mind also the Nambu string in 3 and in 26 dimensions ${ }^{3}$ and the quark-binding string in three dimensions. ${ }^{8}$

(1) If we include some massless scalar particles moving clockwise around the closed string, then the clockwise part of the Lorentz algebra will close if the total number of Dirac and scalar quarks is 22. The anticlockwise part of the Lorentz algebra remains not satisfied.

(2) If we consider the other projection of the quark field, $i \gamma_{5} h h \psi=-\psi$, the quarks will be moving anticlockwise, i.e., $\psi \propto F(\sigma)$.

(3) The spectrum of the subsystem contains the spectrum of the empty string (i.e., Nambu string). ${ }^{3}$ We expect the spectrum of the complete system to contain the spectrum of the closed string with quarks moving clockwise only or with quarks moving anticlockwise only.

(4) The string has only one dimensional parameter $\mathrm{e}$; it is not surprising that the Regge trajectories are straight lines.

(5) For the open string to exist, we need a linear combination of the left- and right-moving quark waves such that the string is a closed system: that is, the energy-momentum and current densities vanish at the ends of the string.

(6) Despite the complicated looking form of the implicit solution of the quark fields, Eq. (3.21), the quark part of the Hamiltonian (4.11) is very simple.

Now we state the speculation:

Conjecture 1. Consider the mass spectrum

$$
\begin{aligned}
\mathfrak{T}^{2}=4 \pi \mathfrak{C} & {\left[\sum_{n>0} a_{n}^{\dagger} \cdot a_{n}+\sum_{j=1}^{N} \sum_{m>0}^{\prime} m\left(b_{j m}^{\dagger} b_{j m}+d_{j m}^{\dagger} d_{j m}\right)\right.} \\
& \left.+\sum_{i=N+1}^{22} s_{n i}^{\dagger} s_{n i}-\alpha_{0}\right]
\end{aligned}
$$

where $a_{n}, s_{n}$ obey the commutator (4.14a); $b_{m}$ and $d_{m}$ obey Eq. (4.13). Its Lorentz algebra can be defined consistently to be identical to that given in Sec. IV with the following modifications

$$
\begin{aligned}
c_{n}= & 0, \quad \tilde{\alpha}_{0}=0, \\
\tilde{\mathscr{L}}_{n}= & 0, \quad E=0, \\
\mathscr{L}_{n}= & \frac{1}{2} \sum_{l}:\left(a_{-l} \cdot a_{n+l}+s_{-l} \cdot s_{n+l}\right): \\
& +: \sum^{\prime}\left(m+\frac{1}{2} n\right) b_{j m}^{\dagger} b_{j, m+n}: .
\end{aligned}
$$

Normal-ordering is with respect to the ground state $|0\rangle$ defined by Eq. (4.15) and

$$
s_{n}|0\rangle=0, \quad n>0
$$

where

$$
d_{m}^{\dagger}=b_{-m},
$$




$$
\begin{aligned}
& d_{m}=b_{-m}^{\dagger}, \\
& s_{-n}=s_{n}^{\dagger} .
\end{aligned}
$$

We interpret $b_{m}$ and $d_{m}$ to be quark and antiquark oscillators, and $s_{n}$ to be massless scalar field oscillators. ${ }^{12}$ Closure of the Lorentz algebra requires $\alpha_{0}=1$. Hence the lowest "mesonic" state $b_{1 / 2}^{\dagger} d_{1 / 2}^{\dagger}|0\rangle$ has zero mass. $\alpha_{0}=1$ also prevents condensation: The mass of a state with $M$ quarkantiquark pairs is heavier than $M$ "mesonic" states each with one of the $M$ quark-antiquark pairs. We speculate that this mass spectrum (and its Lorentz algebra) belongs to the (open) quark-binding string with the addition of $(22-N)$ scalar fields

$$
\mathcal{L} \rightarrow \mathcal{L}+\sqrt{-g}\left(\sum_{i=N+1}^{22} \frac{1}{2} \partial_{\alpha} \phi_{i} \partial^{\alpha} \phi_{i}\right) \text {. }
$$

The Regge slope is $\alpha^{\prime}=1 / 4 \pi \mathbb{e}$ for the open string. We also speculate that the closed quark-binding string has no fermionic states in its spectrum.

\section{B. The quark-confining string}

The quark-binding string model formulated in Sec. II has an arbitrary constant $\mathfrak{C}$ introduced as a tension to prevent the quark fields from expanding without bound. It is the analog of the MIT bay constant (as a pressure) and the SLAC bubble constant (as a surface tension). It may be the effective binding force of some quark-gluon interactions, so that the quark-binding string can be considered as a phenomenological description of some quark-gluon interacting field theory. However, the present formalism is at best preliminary: There is no quark confinement; that is, there are exotic states in the spectrum. Also the presence of pure vibrational modes implies the existence of hadronic states which have no quark modes. Experimentally there is no evidence of such states.

To improve the string model, we present our second conjecture.

Conjecture 2. Consider the following action (without the constant $\mathfrak{e}$ ):

$$
\begin{aligned}
& S=\int d^{2} u^{\alpha} \sqrt{-g}\left[\bar{\psi}_{j} t^{\alpha}\left(\frac{1}{2} i \vec{\partial}_{\alpha}-e A_{\alpha}^{a} T^{a}\right) \psi_{j}-\frac{1}{4} F_{\alpha \beta}^{a} F^{a \alpha \beta}\right] \\
&=\int d^{2} u^{\alpha} \mathcal{L}, \\
& F_{\alpha \beta}^{a}=F_{\mu \nu}^{a} \tau_{\alpha}^{\mu} \tau_{\beta}^{\nu}=\partial_{\alpha} A_{\beta}^{a}-\partial_{\beta} A_{\alpha}^{a}+e f^{a b c} A_{b \alpha} A_{c \beta},
\end{aligned}
$$

and $F_{\alpha \beta}^{a}=0$ at the ends of the string. $A_{\alpha}^{a}=\tau_{\alpha}^{\mu} A_{\mu}^{a}$ are non-Abelian gauge fields in the color SU(3) adjoint representation. Quarks come in color triplets. The index $j$ refers to quark flavors. They must obey the same constraints in Ta- ble I as before to ensure the string is a closed system. Fermion masses $(M \bar{\psi} \psi)$ can of course be added if needed. ${ }^{13} f^{a b c}$ is the group structure constant

$$
\left[T^{a}, T^{b}\right]=f^{a b c} T^{c},
$$

where $T^{a}$ is an SU(3) matrix, $a=1,2, \ldots, 8$. This model we refer to as the quark-confining string, since only color-singlet states exist. If necessary, we can also introduce any number of color-singlet scalar fields into the Lagrangian

$$
\mathcal{L} \rightarrow \mathscr{L}+\sqrt{-g}\left(\frac{1}{2} \partial^{\alpha} \phi_{i} \partial_{\alpha} \phi_{i}-\frac{1}{2} \mu_{i}{ }^{2} \phi_{i}{ }^{2}\right) .
$$

This may be necessary to saturate the Lorentz algebra. Notice that the string constant $\mathfrak{C}$ is not included. Intuitively we see that there is no color vector gluons, since $F_{\alpha \beta}^{a}$ has only one component, namely $F_{01}^{a}=E^{a} ; A_{1}^{a}$ can be removed by an appropriate gauge choice. This leaves only the "Coulomb potential," which provides an attractive force along the string, very much like a tension in the presence of quarks. Hence the spectrum contains color-singlet states with quark and vibrational excitations. In the absence of quarks, the tension (i.e., Coulomb force) also disappears, so that the scalar fields are not bound. Hence one can hope that this relativistic invariant quarkconfining string model has only mesons and baryons among its low-lying states; there are no states which have only vibrational, color, and/or scalar modes. At high energies, there also exist color-singlet states with $3 N^{\prime}$ quarks plus $M$ quarkantiquark pairs. If the Regge intercept is positive, which is probable, these states would have very large widths (to decay to normal baryons and mesons) and therefore would be very difficult to observe.

We believe this quark-confining string, where exact color symmetry and quark-binding (along a line element) are put in by hand, offers enough computational simplifications to make it a worthwhile approach for studying quark confinement.

We may also consider the Han-Nambu color. The color gauge field involved is Abelian. One may hope to solve this model exactly. In practice, it is like putting the Schwinger model in a dynamical curved space, which is embedded in Minkowski space.

It is straightforward to write down the action:

$$
\begin{array}{r}
S=\int d^{2} u^{\alpha} \sqrt{-g}\left[\bar{\psi}_{j} t^{\alpha}\left(\frac{1}{2} i \vec{\partial}_{\alpha}-e_{j} A_{\alpha}\right) \psi_{j}\right. \\
\left.-\frac{1}{4} F^{2}-\mathcal{e}-M_{j} \bar{\psi}_{j} \psi_{j}\right],
\end{array}
$$

where, for the moment, we include the constant e. The equations of motion are [see Fig. 2(a)]

$$
\hbar^{\alpha}\left(i \partial_{\alpha}-e A_{\alpha}\right) \psi=-i\left(k_{1} h+k_{2} \not h\right) \psi+M \psi,
$$




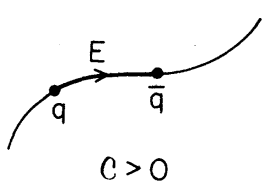

(a)

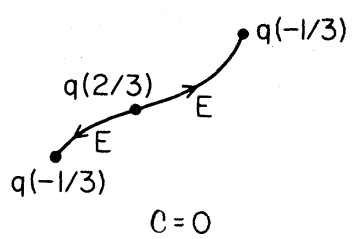

(c)

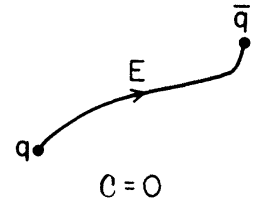

(b)

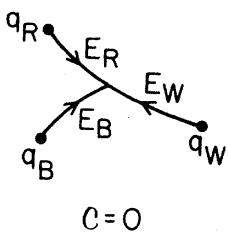

(d)

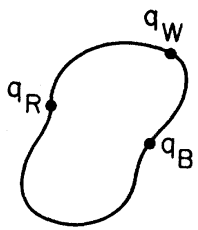

(e)

FIG. 2. Quark-confining strings. In (c), $\frac{2}{3}$ and $\frac{1}{3}$ are the Abelian color hypercharges.

$$
\begin{aligned}
& F_{\| \alpha}^{\alpha \beta}=e_{j} \bar{\psi}_{j} t^{\beta} \psi_{j}, \\
& \begin{aligned}
\left(T^{\alpha \beta} \tau_{\beta \mu}\right)_{\| \alpha}=- & {\left[\bar{\psi}_{j} \not\left(\frac{1}{2} i \ddot{\partial}^{\alpha}-e_{j} A^{\alpha}\right) \psi_{j} n_{\mu}\right.} \\
& \left.+\bar{\psi}_{j} \not h\left(\frac{1}{2} i \ddot{\partial}^{\alpha}-e_{j} A^{\alpha}\right) \psi_{j} m_{\mu}\right]_{\| \alpha},
\end{aligned}
\end{aligned}
$$

where

$$
T^{\alpha \beta}=\left(\mathfrak{e}+\frac{1}{4} F^{2}\right) g^{\alpha \beta}+\bar{\psi}_{j} t^{\alpha}\left(\frac{1}{2} i \ddot{\partial}^{\beta}-e_{j} A^{\beta}\right) \psi_{j}-F^{\alpha \sigma} F_{\sigma}^{\beta}
$$

and $j$ is the index for quark types. Neglecting the quark term in $T^{\alpha \beta}$, we see that, in the coordinate

$$
\begin{aligned}
g_{\alpha \beta} & =\left(\begin{array}{cc}
1 & 0 \\
0 & -1
\end{array}\right), \\
T^{00} & =\mathfrak{C}+\frac{1}{2} E^{2} \\
& =\mathfrak{e}+\frac{1}{2}\left(F^{01}\right)^{2}>0 .
\end{aligned}
$$

Since the electric field $\left(E^{2}\right)$ term is positive, we can take $\mathbf{C} \rightarrow 0$. The boundary conditions at the ends of the string must be imposed to ensure the system is closed:

$$
\begin{aligned}
& E=0, \text { at ends } \\
& P_{\mu}^{\circ}=0 .
\end{aligned}
$$

This means each end of the string must end with a quark (or an antiquark) as in Fig. 2(b). The bary- on string has a configuration shown in Fig. 2(c). For the non-Abelian case, Figs. 2(d) and 2(e) may also have to be considered. The coupling constant $e$ is the only dimensional parameter in the model in the absence of the constant $\mathcal{C}$ and masses $M$; $\left(e_{j} \propto e\right)$. In the presence of quark masses, the Regge trajectories will not be linear for the lowmass states.

For the case where $(M / e)^{2} \ll 1$, the system should be solved in the absence of masses and the mass terms should be treated perturbatively. For the case $(M / e)^{2} \gg 1$, it is straightforward to show (and is left as an exercise for the reader) that, to the lowest-order approximation, the potential between a quark and an antiquark rises linearly $V(r) \propto r$.

\section{ACKNOWLEDGMENTS}

The author would like to thank his colleagues at SLAC, especially R. Giles, for helpful discussions.

\section{APPENDIX A}

The best way to show $j_{i} \psi_{k}=0$ is probably the straightforward way. Let

$$
\psi=\left[\begin{array}{l}
u \\
v \\
w \\
z
\end{array}\right] .
$$

Equation (2.20) puts constraints among the four components. Using the Dirac representation (2.15) the constraints become

$$
\begin{aligned}
& v=g u=\frac{1+i\left(m_{l} n_{r}-m_{+} n_{-}\right)}{i\left(m_{l} n_{+}-m_{+} n_{l}\right)} u, \\
& z=f w=\frac{1+i\left(m_{-} n_{+}-m_{l} n_{r}\right)}{i\left(n_{-} m_{l}-m_{-} n_{l}\right)} w,
\end{aligned}
$$

where

$$
\begin{aligned}
& m_{ \pm}=m_{0} \pm m_{3}, \\
& m_{r}=m_{1}+i m_{2}=m_{l}^{*},
\end{aligned}
$$

and similarly for $n_{ \pm}$and $n_{l, r}$. Using Eqs. (2.6) and (2.7), we obtain

$$
f^{\dagger} g=f g^{\dagger}=-1
$$

Now

$$
\begin{aligned}
j & =\left(\begin{array}{cc}
0 & -j_{0}-\vec{j} \cdot \vec{\sigma} \\
-j_{0}+\vec{j} \cdot \vec{\sigma} & 0
\end{array}\right) \\
& =\left(\bar{\psi} \gamma_{\mu} \psi\right) \gamma^{\mu} .
\end{aligned}
$$


Hence

$$
\dot{j} \psi_{k}=\left[\begin{array}{c}
-j_{+} w_{k}-j_{l} z_{k} \\
-j_{r} w_{k}-j_{-} z_{k} \\
-j_{-} u_{k}+j_{l} v_{k} \\
j_{r} u_{k}-j_{+} v_{k}
\end{array}\right] \propto 1+f^{\dagger} g=0
$$

where

$$
\begin{aligned}
& j_{+}=2 u^{\dagger} u+2 z^{\dagger} z, \\
& j_{-}=2 v^{\dagger} v+2 w^{\dagger} w, \\
& j_{l}=2 v^{\dagger} u-2 z^{\dagger} w, \\
& j_{r}=2 u^{\dagger} v-2 w^{\dagger} z,
\end{aligned}
$$

where $\psi\left(\right.$ in $\left.j_{\mu}\right)$ and $\psi_{k}$ can either be the same spinor or different spinors.

\section{APPENDIX B}

$T^{\alpha \beta}$ can be chosen to be off-diagonal and symmetric if it is symmetric and has signature $(1,-1)$ to begin with. The proof is almost identical to that given for Ref. 8. Since $g^{\alpha \beta}$ is already symmetric, we need to show $\theta^{\alpha \beta}$ is symmetric. $\theta^{\alpha \beta}$ is a tensor; hence it is sufficient to show that it is symmetric at any given point in some coordinate system. At any given point, we can choose locally geodesic coordinates

$$
\begin{aligned}
& g_{\alpha \beta}=\left(\begin{array}{cc}
1 & 0 \\
0 & -1
\end{array}\right), \\
& g_{\alpha \beta \mid \gamma}=0 .
\end{aligned}
$$

Equation (3.2) becomes

$$
t^{1} t^{0}=-i \gamma_{5} \operatorname{mh} h=-t^{0} t^{1} .
$$

It is then straightforward to show that $\theta^{01}=\theta^{10}$ in this coordinate system using the Dirac equation (2.25) and Eq. (2.21). To show that $T^{\alpha \beta}$ has signature $(1,-1)$ all we need is to show that $\operatorname{det}\left(T^{\alpha \beta}\right)$ $<0$, where

$$
\operatorname{det}\left(T^{\alpha \beta}\right)=\frac{e^{2}}{g}+\frac{e}{g} \theta^{\alpha} \alpha+\operatorname{det}\left(\theta^{\alpha \beta}\right) .
$$

Dirac equation (2.25) implies

$$
\frac{1}{2} i \bar{\psi} t^{\alpha} \vec{\partial}_{\alpha} \psi=0 \text {, }
$$

and the lightlike currents (Appendix A) imply the vanishing of the last term. Since $g^{\alpha \beta}$ also has signature $(1,-1)$ then

$$
\operatorname{det}\left(T^{\alpha \beta}\right)=\frac{\mathrm{e}^{2}}{g}<0 \text {. }
$$

That $T^{\alpha \beta}$ can be chosen to be off-diagonal is, of course, due to the arbitrariness in the choice of the parametrization. This is also related to the fact that our phase space is constrained. To see this, let us consider the canonical momentum $\mathbb{P}_{\mu}^{0}=\delta \mathscr{L} / \delta \dot{x}^{\mu}$ and the shifted coordinate

$$
y_{1 \mu}=\tau_{1 \mu}-\frac{1}{2 \mathfrak{e}} i \bar{\psi}_{j} \gamma_{\mu} \vec{\partial}_{1} \psi_{j}
$$

Naively one may expect them to be independent. However, they are constrained by the following relations:

$$
\begin{aligned}
& \boldsymbol{P}^{0} \cdot y_{1}=0, \\
& \boldsymbol{P}_{\mu}^{0} \boldsymbol{P}^{0 \mu}+\mathbb{C}^{2} y_{1 \mu} y^{1 \mu}=0 .
\end{aligned}
$$

\section{APPENDIX C}

The Gauss-Codazzi equations involve the torsion $v_{\alpha}$ which depends on the choice of the normals $n_{\mu}$ and $m_{\mu}$. To verify the Gauss-Codazzi equations in the coordinates chosen, we first make an explicit choice of $n_{\mu}$ and $m_{\mu}$; we then calculate all the curvature tensors and the torsion and substitute them directly into the equations.

The normal plane

$$
U_{\mu \nu}=n_{\mu} m_{\nu}-m_{\mu} n_{\nu}
$$

is invariant under the rotation

$$
\begin{aligned}
& n_{\mu} \rightarrow n_{\mu} \cos \theta+m_{\mu} \sin \theta, \\
& m_{\mu} \rightarrow-n_{\mu} \sin \theta+m_{\mu} \cos \theta .
\end{aligned}
$$

Let us choose $n_{\mu}$ such that $n_{+}=n_{0}+n_{3}=0$. (This is a valid choice since $n_{\mu}$ is spacelike.) To do so, it is convenient to introduce some notations. Explicitly Eq. (2.4) gives

$$
\begin{aligned}
& a=V U_{+-}=2\left(\dot{x}_{2} x_{1}^{\prime}-\dot{x}_{1} x_{2}^{\prime}\right), \\
& b=V U_{+1}=\dot{x}_{2} x_{+}^{\prime}-\dot{x}_{+} x_{2}^{\prime}=p\left(\dot{x}_{2}-x_{2}^{\prime}\right)=p s, \\
& c=V U_{+2}=\dot{x}_{+} x_{1}^{\prime}-\dot{x}_{1} x_{+}^{\prime}=p\left(x_{1}^{\prime}-\dot{x}_{1}\right)=-p t, \\
& d=V U_{12}=\frac{1}{2}\left(\dot{x}_{+} x_{-}^{\prime}-\dot{x}_{-} x_{+}^{\prime}\right)=\frac{1}{2} p\left(x_{-}^{\prime}-\dot{x}_{-}\right), \\
& e=V U_{-1}=\dot{x}_{-} x_{2}^{\prime}-\dot{x}_{2} x_{-}^{\prime}, \\
& f=V U_{-2}=\dot{x}_{1} x_{-}^{\prime}-\dot{x}_{-} x_{1}^{\prime},
\end{aligned}
$$

where

$$
V=\sqrt{-g}
$$

Note that

$$
b f=a d+c e
$$

and

$$
\begin{aligned}
V & =\frac{t^{2}+s^{2}+A}{2} \\
& =d^{2}-\frac{1}{4} a^{2}-b e-c f .
\end{aligned}
$$

Now it is straightforward to construct $n_{\mu}, m_{\mu}$ in 
terms of $\dot{x}_{\mu}, x_{\mu}^{\prime}$ :

$$
\begin{aligned}
n_{\mu}= & \left(n_{+}, n_{-}, n_{1}, n_{2}\right) \\
= & \frac{1}{\left(b^{2}+c^{2}\right)^{1 / 2}}(0, a, b, c), \\
m_{\mu}= & \frac{1}{2 V\left(b^{2}+c^{2}\right)^{1 / 2}} \\
& \times\left(2 b^{2}+2 c^{2}, a^{2}+2 b e+2 c f, b a+2 c d, a c-2 b d\right) .
\end{aligned}
$$

Using Eq. (3.11), we now obtain

$$
\begin{aligned}
& l_{11}=\frac{t x_{1}^{\prime \prime}+s x_{2}^{\prime \prime}}{\left(s^{2}+t^{2}\right)^{1 / 2}}, \\
& h_{11}=\frac{s x_{1}^{\prime \prime}-t x_{2}^{\prime \prime}}{\left(s^{2}+t^{2}\right)^{1 / 2}}, \\
& \nu_{0}=-\left.m_{\mu} n^{\mu}\right|_{0}=\frac{t \ddot{x}_{2}-s \ddot{x}_{1}}{s^{2}+t^{2}}, \\
& \nu_{1}=\left(\frac{1}{s^{2}+t^{2}}-\frac{1}{V}\right)\left(s x_{1}^{\prime \prime}-t x_{2}^{\prime \prime}\right) .
\end{aligned}
$$

It is clear that the second Gauss-Codazzi equation (3.15) is satisfied.

The first Gauss-Codazzi equation (3.14) can also be verified easily once we obtain the other components of the curvature tensors. They are, using Eq. (3.10),

$$
\begin{aligned}
& h_{00}=\frac{s \ddot{x}_{1}-t \ddot{x}_{2}^{\circ}}{\left(s^{2}+t^{2}\right)^{1 / 2}}, \\
& l_{00}=\frac{1}{V\left(s^{2}+t^{2}\right)^{1 / 2}}\left(A \dot{V}-\frac{1}{2} \dot{A} V-\dot{V} V\right),
\end{aligned}
$$

where

$$
\dot{V}=\frac{1}{2} \dot{A}+t \ddot{x}_{1}+s \ddot{x}_{2} .
$$

For the record, we note that the "mean curvature" is given by

$$
\begin{aligned}
k & =\left(k_{1}^{2}+{k_{2}}^{2}\right)^{1 / 2} \\
& =-\frac{A}{2 V^{2}}\left(x_{1}^{\prime \prime 2}+x_{2}^{\prime \prime 2}\right)^{1 / 2} .
\end{aligned}
$$

\section{APPENDIX D}

Given the commutators among the independent operators $a_{n}, c_{n}, b_{m}, d_{m}, q_{i}, q_{-}, a_{0}^{i}$, and $a_{0}^{+}$, all commutators can be calculated. We give some of the useful ones.

$$
\begin{aligned}
{\left[Q_{j}, b_{i m}^{\dagger}\right] } & =\delta_{i j} b_{i m}^{\dagger}, \\
{\left[Q_{j}, d_{i m}^{\dagger}\right] } & =-\delta_{i j} d_{i m}^{\dagger}, \\
{\left[\mathscr{L}_{n}, a_{l}\right] } & =-l a_{l+n}, \\
{\left[\mathscr{L}_{n}, b_{m}\right] } & =-\left(m+\frac{1}{2} n\right) b_{m+n}, \\
{\left[\mathscr{L}_{n}, b_{m}^{\dagger}\right]=} & \left(m-\frac{1}{2} n\right) b_{m-n}^{\dagger}, \\
{\left[\tilde{\mathscr{L}}_{n}, c_{l}\right]=} & -l c_{l+n}, \\
{\left[q_{-}, \frac{1}{a_{0}^{+}}\right]=} & \frac{i}{\left(a_{0}^{+}\right)^{2}}, \\
{\left[\mathcal{L}_{n}, \mathscr{L}_{m}\right]=} & (n-m) \mathscr{L}_{n+m} \\
& +\delta_{n,-m}\left(n^{3}-n\right) \frac{\left(\frac{1}{2} N+1\right)}{12}, \\
{\left[\tilde{\mathscr{L}}_{n}, \tilde{\mathscr{L}}_{m}\right]=} & (n-m) \tilde{\mathscr{L}}_{n+m} \\
& +\delta_{n,-m} \frac{\left(n^{3}-n\right)}{12},
\end{aligned}
$$

where $N$ is the number of quark fields. The constant terms in the last two commutators play the same role as $c$-number Schwinger terms.
*Work supported by the U. S. Energy Research and Development Administration.

${ }^{1} \mathrm{Y}$. Nambu, in Symmetries and Quark Models, edited by R. Chand (Gordon and Breach, New York, 1970); H. Nielsen, in High Energy Physics, Proceedings of the Fifteenth International Conference on High Energy Physics, Kiev, 1970, edited by V. Shelest (Naukova Dumka, Kiev, USSR, 1972); L. Susskind, Nuovo Cimento 69A, 457 (1970); T. Takabayashi, Prog. Theor. Phys. $\underline{43}, 1117$ (1970).

${ }^{2}$ G. Veneziano, Nuovo Cimento 57A, 190 (1968), and numerous papers thereafter.

${ }^{3} \mathrm{Y}$. Nambu, lectures at Copenhagen Summer Symposium, 1970 (unpublished); P. Goddard, J. Goldstone, C. Rebbi, and C. B. Thorn, Nucl. Phys. B56, 109 (1973). See also L.-N. Chang and F. Mansouri, Phys. Rev. D $\underline{5}$, 2535 (1972).

${ }^{4}$ See, e.g., H. B. Nielsen and P. Olesen, Nucl. Phys. B61, 45 (1973); K. Wilson, Phys. Rev. D 10, 2445

(1974); A. M. Polyakov, Landau Institute report, 1974 (unpublished); Y. Nambu, Phys. Rev. D 10, 4262 (1974); J. Kogut and L. Susskind, ibid. 11, 395 (1975); S. Mandelstam, Phys. Lett. 53B, 476 (1975); L. D. Faddeev, Max-Planck-Institut (München) Report No. MPI-PAE/ Pth 16, 1974 (unpublished); Z. F. Ezawa and H. C. Tze, Nucl. Phys. B100, 1 (1976).

${ }^{5}$ S. Mandelstam, Nucl. Phys. B64, 205 (1973).

${ }^{6}$ A. Chodos, R. L. Jaffe, K. Johnson, C. B. Thorn, and V. F. Weisskopf, Phys. Rev. D $\underline{9}, 3471$ (1974).

${ }^{7}$ W. A. Bardeen, M. S. Chanowitz, S. D. Drell, M. Weinstein, and T.-M. Yan, Phys. Rev. D 11, 1094 (1975); R. C. Giles, ibid. 13,1670 (1976).

${ }^{8}$ R. C. Giles and S. - H. H. Tye, Phys. Rev. D $\underline{13}, 1690$ (1976).

${ }^{9}$ See e.g. L. P. Eisenhart, Riemannian Geometry (Princeton Univ. Press, Princeton, New Jersey, 1949). See R. C. Giles, Ref. 7, for further references and also a lucid discussion on the embedding of hypersurfaces.

${ }^{10}$ After the completion of this work we were informed by L.-N. Chang of the work by L.-N. Chang and F. Man- 
souri in Workshop on Current Problems in High Energy Physics, Johns Hopkins University, 1974 (unpublished), where a similar model has been considered.

${ }^{11}$ R. Staffin (unpublîshed).

${ }^{12}$ Compare with K. Bardakci and M. B. Halpern, Phys. Rev. D 3, 2493 (1971).
${ }^{13}$ The constraint $\bar{\psi} \psi=0$ must be relaxed in the presence of quark mass. The derivation of the equations of motion does not use it. Quark masses also require further relaxation of the constraints on the fermion fields. 